\title{
Comparison of trunk muscle activity according to hip abduction angle during plank exercise
}

\author{
Tae jin $\mathrm{Pi}^{\mathrm{a}}$, Minkwon Cho ${ }^{\mathrm{b}}$, Suyoung Shim ${ }^{\mathrm{c}}$, Jongchan Jung ${ }^{\mathrm{c}}$, Yijung Chung ${ }^{\mathrm{d}}$ \\ ${ }^{a}$ Department of Physical Therapy, SOL Hospital, Seoul, Republic of Korea \\ ${ }^{b}$ Department of Physical Therapy, Kaiser Hospital, Guri, Republic of Korea \\ 'Department of Physical Therapy, The Graduate School, Sahmyook University, Seoul, Republic of Korea \\ ${ }^{d}$ Department of Physical Therapy, College of Health Science and Social Welfare, Sahmyook University, Seoul, Republic of Korea
}

Objective: The purpose of this study was to investigate the conditions for the application of various plank exercises to people who require trunk stabilization by comparing trunk muscle activity according to the degree of hip abduction in the plank exercise.

Design: Cross-sectional study.

Methods: Twenty healthy participants voluntarily participated in this study and the plank exercise was performed under 5 conditions (two-legged support plank [TSP] with hip abduction, TSP with hip abduction of 15 degrees, TSP with hip abduction of 30 , one-legged support plank [OSP] with hip abduction of 15 degrees, OSP with hip abduction of 30 degrees). In order to measure the trunk muscle activity according to the 5 conditions, surface electromyography was used. The electrical activities of the rectus abdominis (RA), external oblique (EO), and internal oblique (IO) muscles were measured during the 5 plank exercises. Subjects practiced each of the 5 conditions three times in random order and the average values were obtained.

Results: In the OSP condition with 15/30 degrees of hip abduction, activities of the RA, EO, and IO were significantly greater than during the TSP $(p<0.05)$. In the OSP with 30 degrees of hip abduction condition, activities of the left EO, IO were significantly greater than other plank exercise conditions $(p<0.05)$.

Conclusions: The plank exercise with hip abduction of 30 degrees and the OSP exercise can be suggested as an effective method to enhance the activity of the trunk oblique muscles.

Key Words: Abdominal muscles, Electromyography, Hip joint, Plank exercise

\section{Introduction}

Trunk stabilization exercises are one of the important components of rehabilitation training for increasing trunk muscle activation [1] because it is possible to re-educate the trunk muscles for proper activation and coordination through improved exercise control, and they can lead to improvement of vertebral stability [2,3]. It also maintains spinal alignment, provides adequate motion control for functional activity, and is widely used to prevent and treat back pain [4].

The development of core muscles is important in many functional activities because it improves trunk stability, pro- vides proximal stability of the body, and facilitates distal movement [5]. The core muscles include various muscles, such as the rectus abdominis (RA), internal and external obliques (EOs), and the multifidus muscles. Since they are involved in controlling the the neutral position of the vertebrae during dynamic functional activities, it is important perform exercises to increase the activity of the internal and EO muscle activity [6].

Plank exercises are popular for strengthening the core muscles, including internal and EO muscles [7], and is a passive and active stabilizer of the lumbar pelvis region that maintains proper balance and control of the trunk and hip

Received: 13 March, 2019 Revised: 13 May, 2019 Accepted: 16 May, 2019

Corresponding author: Yijung Chung (ORCID https://orcid.org/0000-0002-2431-8895)

Department of Physical Therapy, College of Health Science and Social Welfare, Sahmyook University, Seoul, Republic of Korea Tel: 82-2-3399-1637 Fax: 82-2-3399-1639 E-mail: yijung36@syu.ac.kr

(c) This is an Open-Access article distributed under the terms of the Creative Commons Attribution Non-Commercial License (http://creativecommons.org/licenses/ by-nc/4.0) which permits unrestricted non-commercial use, distribution, and reproduction in any medium, provided the original work is properly cited.

Copyright (๑ 2019 Korean Academy of Physical Therapy Rehabilitation Science 
joint during both static and dynamic movement of the body [8]. Sit-ups, crunches, and plank movements spinal stabilization exercises that are effective for strengthenening the core muscles without time and space constraints [9]. When comparing sit-up exercises and plank exercises, which have been widely used as stabilizing exercises for the trunk, sit-up exercises increase the compression force on the vertebrae, making it easy to cause degenerative spinal injuries [10]. On the other hand, plank exercise is an optimal exercise to increase abdominal muscle strength because the lumbar pelvic region can be placed in a neutral position to reduce the load placed onto the vertebrae [7].

There have recent been studies on modified plank motions with the addition of limb movements.

Kim et al. [6] reported increased activity of the RA and internal and EO muscles in the plank exercise with isometric contraction of the hip adduction muscles. Schoenfeld et al. [11] reported increased rectus abdominus and EO muscle activity during the modified plank exercise. In addition, when the posture with hip abduction was maintained in the stance position, the activity of the anterior muscles of the trunk was greater on an unstable surface than in the stable basal plane [12]. As shown in the above study, the addition of limb movements to the trunk muscle stabilization exercises further increased abdominal activation [13], and in particular, the movement of one limb further increased the activity of the abdominal muscles associated with trunk stability [14]. Although there are several studies that show that plank exercises are effective, there are few studies that have investigated the activation of trunk muscles during plank exercises according to postural changes [9], and studies with limb movements during modified plank exercise are also lacking.

In particular, there are few studies that have investigated trunk muscle activity during plank exercises according different hip abduction angles. Therefore, the purpose of this study was to investigate the activation of the trunk muscles when the abduction angle of one hip joint was differentiated during the plank exercise and to provide basic information on effective plank exercise methods.

\section{Methods}

\section{Participants}

This study was conducted on adult males and females (16 males and 4 females) working in SOL hospital who understood the study and expressed their intention to participate actively. This study was approved by the research Sahmyook University Life Science ethics committee (IRB No. 2-7001793-AB-N-012019006HR) and informed consent was obtained.

The subjects of the study were selected as those who had no musculoskeletal or nervous system or cardiovascular disease and symptoms in the trunk, upper limb, and lower limb during the past 6 months and did not complain of shoulder pain and back pain in performing the plank exercise posture, those who did not perform extreme exercise the day before the experiment, and healthy adults who did not have any accompanying diseases that would restrict other research activities. The exclusion criteria were those medically diagnosed with back and upper limb damage, those who could not be tested because of musculoskeletal injuries, those who have been diagnosed with medical problems that would restrict them from exercising, and those who have consumed alcohol on the day before and the day of the experiment (Table 1).

\section{Procedures}

The maximum voluntary isometric contraction (MVIC) was measured after attaching electrodes to both the RA and the external and internal oblique (IO) muscles of the subjects [9]. Prior to the experiment, the researchers selected the hip joint abduction site of the lower limb. After measuring 0,15 , and 30 degrees of hip abduction with a goniometer on the mat to be tested for each subject, the position of the subjects'left leg was indicated by markers at the corresponding angle point, and the position of the right leg placed at the level of the right end of the mat. Prior to the experiment, the researchers ensured that the subjects were able to understand the experimental method by providing explanation about the plank posture and allowing the subject so practice each plank more than 3 times [20]. The plank exercises were performed in 5 different postures randomly, with each repeated 3 times.

Table 1. General characteristics of the subjects $(\mathrm{N}=20)$

\begin{tabular}{lr}
\hline \multicolumn{1}{c}{ Characteristic } & \multicolumn{1}{c}{ Subjects } \\
\hline Sex (male/female) & $20(16 / 4)$ \\
Age $(\mathrm{y})$ & $27.75(1.62)$ \\
Height $(\mathrm{cm})$ & $173.20(5.69)$ \\
Weight $(\mathrm{kg})$ & $66.25(7.89)$ \\
\hline
\end{tabular}

Values are presented as number only or mean (SD). 


\section{Two-legged support plank with $0^{\circ}$ of hip abduction}

In the prone position, the elbows and forearms of the upper limbs were placed on the floor and were at right angles with the shoulders. The legs were fully extended without any hip abduction and with only the tip of the feet touching the floor (Figure 1A) [6,9].

\section{Two-legged support plank with $15^{\circ}$ of hip abduction}

In a basic plank posture, such as a two-legged support plank (TSP), the right leg remained intact and the left hip was abducted to $15^{\circ}$ (Figure $1 \mathrm{~A}$ ).

\section{Two-legged support plank with $30^{\circ}$ of hip abduction}

In a basic posture, such as a TSP, the right leg remained intact and the left hip was abducted to $30^{\circ}$ (Figure 1A).

\section{One-legged support plank with $15^{\circ}$ of hip abduction}

In the TSP position, the right leg was in contact with the floor while the left leg was raised up from the floor with the hip $15^{\circ}$ of abduction (Figure $1 \mathrm{~B}$ ).

\section{One-legged support plank with $30^{\circ}$ of hip abduction}

In the TSP position, the right leg was in contact with the floor while the left leg is raised up from the floor with the hip in $30^{\circ}$ of abduction (Figure 1B).

All subjects were instructed to perform the 5 different flank positions and to maintain each position for 5 seconds. Each plank condition was repeated three times and the subjects were allowed to take one-minute rest between each condition. The experiment was carried out in random order [20].

\section{Measurement}

\section{Collection of electromyography data}

Muscle electromyography (EMG) signals measured in this study were processed by full wave rectification using the Myoresearch XP Master edition software (2013; Noraxon Inc., Scottsdale, AZ, USA) and the root mean square. The maximum voluntary contraction (MVC) value was calculated by dividing the MVIC value by the percent MVC value [9]. Each plank posture was maintained for 5 seconds but data was analyzed during a stable period of 3 seconds with the first and last second extracted, and after the average values were obtained from repeating the measurement three times in all conditions, data was normalized to MVIC values $[6,20]$. To investigate muscle activity during the plank exercise, the surface EMG Telemyo DTS EMG system (2013; Noraxon Inc.) was used. The sampling rate of the EMG signal was set to $100 \mathrm{~Hz}$ and the frequency bandwidth was 10 to $450 \mathrm{~Hz}$ [20].

The electrodes were attached to the RA muscle at the site $2 \mathrm{~cm}$ away from the navel, between the iliac crest and the rib for the EO, and inward and downward from the anterior superior iliac spine (ASIS) $2 \mathrm{~cm}$ apart from each other for the IO [15]. In order to minimize skin resistance, leg hair was removed from the electrode site with a disposable razor before attaching the electrode, and the electrode was cleaned with alcohol to remove the oil or foreign matter [9].

Prior to the experiment, MVIC measurements were performed to obtain the contraction values of the RA, the EO, and the IO muscles for 5 seconds. For the RA, external and IO muscles, the MVIC was obtained by having the subject start in the supine position, and with the therapist fixating the

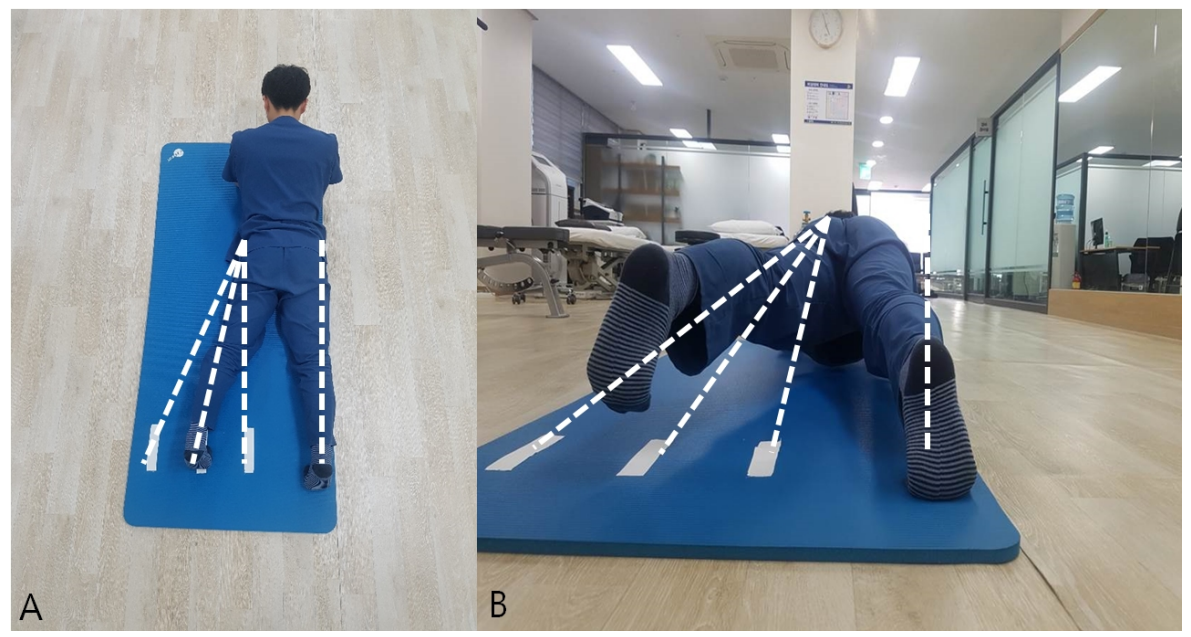

Figure 1. Plank exercise. (A) Two-legged support plank with hip abduction. (B) One-legged support plank with hip abduction. 
Table 2. Comparison of activities of trunk muscle in plank exercise with hip abduction

\begin{tabular}{|c|c|c|c|c|c|c|}
\hline \multirow{2}{*}{ Condition } & \multicolumn{2}{|c|}{ Rectus abdominis } & \multicolumn{2}{|c|}{ External oblique } & \multicolumn{2}{|c|}{ Internal oblique } \\
\hline & Right & Left & Right & Left & Right & Left \\
\hline TSP with $\mathrm{HAB} 0\left(^{\circ}\right)$ & $18.20(8.48)$ & $18.35(7.17)$ & $25.78(8.72)$ & $27.18(12.49)$ & $19.50(7.25)$ & $19.13(7.77)$ \\
\hline TSP with HAB15( $\left(^{\circ}\right)$ & $19.66(8.08)$ & $20.31(8.04)^{\mathrm{a}}$ & $29.88(11.67)^{\mathrm{a}}$ & $31.40(14.22)^{\mathrm{a}}$ & $25.06(10.60)^{\mathrm{a}}$ & $24.10(10.45)^{\mathrm{a}}$ \\
\hline TSP with HAB30 $\left(^{\circ}\right)$ & $23.18(9.53)^{\mathrm{a}, \mathrm{b}}$ & $22.82(8.38)^{\mathrm{a}, \mathrm{b}}$ & $32.47(13.00)^{\mathrm{a}, \mathrm{b}}$ & $34.43(16.71)^{\mathrm{a}, \mathrm{b}}$ & $29.52(11.63)^{\mathrm{a}, \mathrm{b}}$ & $27.35(12.68)^{\mathrm{a}, \mathrm{b}}$ \\
\hline OSP with $\mathrm{HAB} 15\left(^{\circ}\right)$ & $25.92(14.87)^{\mathrm{a}, \mathrm{b}}$ & $25.34(14.83)^{\mathrm{a}}$ & $34.62(15.57)^{\mathrm{a}, \mathrm{b}}$ & $36.72(14.97)^{\mathrm{a}, \mathrm{b}}$ & $32.57(15.52)^{\mathrm{a}, \mathrm{b}}$ & $32.25(15.41)^{\mathrm{a}, \mathrm{b}}$ \\
\hline OSP with $\mathrm{HAB} 30\left(^{\circ}\right)$ & $27.97(15.07)^{\mathrm{a}, \mathrm{b}, \mathrm{d}}$ & $27.26(15.11)^{\mathrm{a}, \mathrm{b}, \mathrm{d}}$ & $35.55(15.32)^{a, b, d}$ & $41.27(14.59)^{\mathrm{a}, \mathrm{b}, \mathrm{c}, \mathrm{d}}$ & $35.70(17.26)^{a, b, d}$ & $34.75(16.51)^{\mathrm{a}, \mathrm{b}, \mathrm{c}, \mathrm{d}}$ \\
\hline $\mathrm{F}(p)$ & $7.29(0.001)$ & $9.51(0.001)$ & $10.92(0.001)$ & $5.08(0.001)$ & $14.35(0.001)$ & $15.99(0.001)$ \\
\hline
\end{tabular}

Values are presented as mean (SD).

TSP: two-legged support plank, HAB: hip abduction, OSP: one-legged support plank.

${ }^{\mathrm{a}}$ Statistically significant difference with TSP with HAB0 $(p<0.05)$.

${ }^{\mathrm{b}}$ Statistically significant difference with TSP with HAB15 $(p<0.05)$.

${ }^{\mathrm{c}}$ Statistically significant difference with TSP with HAB30 $(p<0.05)$.

${ }^{\mathrm{d}}$ Statistically significant difference with OSP with HAB15 $(p<0.05)$.

thighs the subject lifted the upper body so that the spine of the scapula was not in contact with the floor. For the internal and EOs muscles, subjects were placed in the testing posture of the rectus abdomins with the addition of rotational resistance [16].

\section{Data and statistical analysis}

The collected data were analyzed using IBM SPSS Statistics for Windows, Version 19.0 (IBM Co., Armonk, NY, USA). The general characteristics of the subjects were analyzed by descriptive statistics and one-way repeated measures ANOVA was performed to investigate the effect of performing the plank exercises with various hip joint abduction angles on trunk muscle activity. The least significant difference was used for post-test to see if there were any differences in the muscle activity between the plank conditions. All data were statistically significant at $p<0.05$.

\section{Results}

\section{Comparison of bilateral rectus abdominis muscle activity}

The TSP with hip abduction (HAB)30 condition showed significantly greater muscle activity than TSP with HAB0 and TSP with HAB15 plank conditions $(p<0.05)$. In one-legged support plank (OSP) with HAB15, the muscle activity of right RA muscle was significantly greater than TSP with HAB0 and TSP with HAB15 plank conditions $(p<0.05)$. However, there was no significant difference compared with TSP with HAB30. In the OSP with HAB30, the TSP with HAB0, TSP with HAB15, and OSP with HAB15 had significantly greater muscle activity than the right RA muscle $(p<0.05)$ (Table 2)

However, there was no significant difference when comparing TSP with HAB30. In TSP with HAB15, the muscle activity of the left rectus abdominus muscle was significantly larger than that of TSP with HAB $0(p<0.05)$. The TSP with HAB0, TSP with HAB0, and TSP with HAB15 showed significantly greater muscle activity than the TSP with HAB0 $(p<0.05)$. In OSP with HAB15, the left RA muscle activity was significantly greater than TSP with HAB0 $(p<0.05)$. However, there was no significant difference between TSP with HAB15 and TSP with HAB30. In OSP with HAB30, the muscle activity of the left lateral RA muscle was significantly greater than that of TSP with HAB0, TSP with HAB15 and OSP with HAB15 plank conditions $(p<0.05)$ (Figure 2, Table 2).

\section{Comparison of bilateral external oblique muscle activity}

The TSP with HAB15 showed significantly greater bilateral EO muscle activity than the TSP with $\mathrm{HAB} 0(p<0.05)$. The TSP with HAB30, TSP with HAB0, and TSP with HAB 15 showed significantly greater bilateral EO muscle activity than the TSP with HAB30 $(p<0.05)$. In OSP with HAB15, bilateral EO muscle activity was significantly greater than TSP with HAB0 and TSP with HAB15 $(p<0.05)$. However, there was no significant difference compared with TSP with HAB30. In OSP with HAB30, TSP with HAB0, TSP with HAB15, and OSP with HAB15 showed significantly greater activity of right EO muscle $(p<0.05)$ than TSP with HAB0, TSP with HAB15 and OSP with HAB15 $(p<0.05)$, respectively (Figure 3, Table 2). 


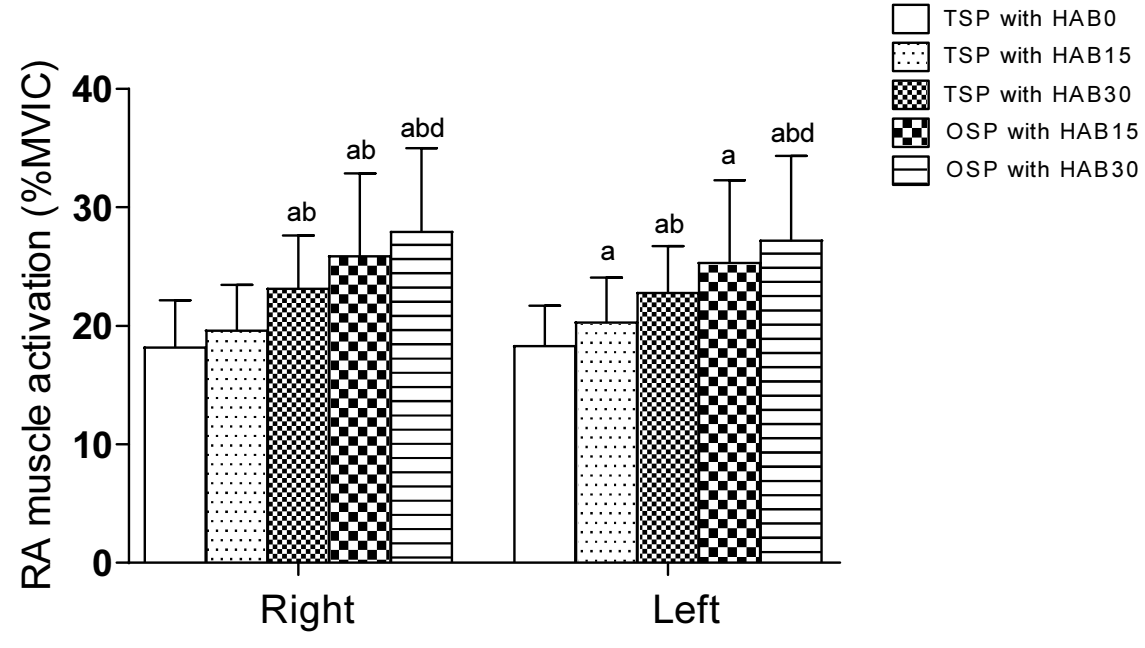

TSP=Two leg support plank; OSP=One leg support plank; HAB=Hip abduction.

aStatistically significant difference with TSP with HABO $(p<0.05)$.

bStatistically significant difference with TSP with HAB15 $(p<0.05)$.

'Statistically significant difference with TSP with HAB30 $(p<0.05)$.

'Statistically significant difference with OSP with HAB15 $(p<0.05)$.

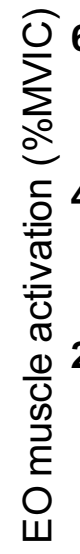
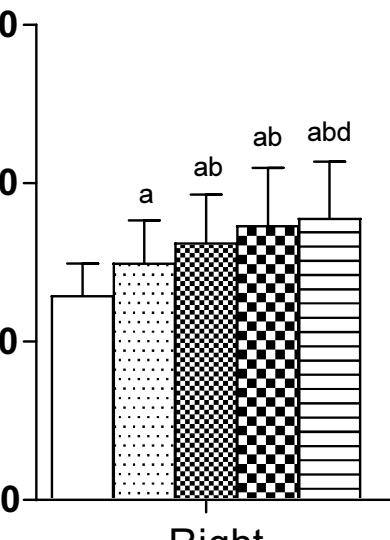

Right

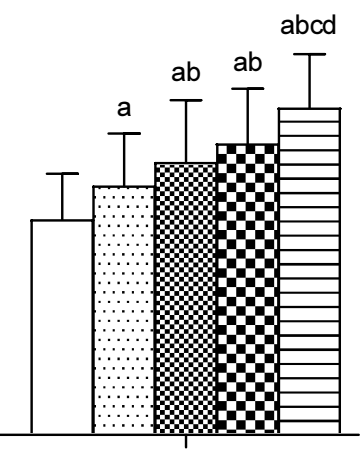

Left

$T S P=T$ wo leg support plank; OSP=One leg support plank; $\mathrm{HAB}=$ Hip abduction

${ }^{\text {aS }}$ Statistically significant difference with TSP with HABO $(p<0.05)$.

${ }^{b}$ Statistically significant difference with TSP with HAB15 $(p<0.05)$.

${ }^{\mathrm{C} S}$ Statistically significant difference with TSP with HAB30 $(p<0.05)$.

${ }^{d}$ Statistically significant difference with OSP with HAB15 $(p<0.05)$.
Figure 2. Rectus abdominis (RA) muscle activities of 5 conditions. MVIC: maximum voluntary isometric contraction.
Figure 3. External oblique (EO) muscle activities of 5 conditions. MVIC: maximum voluntary isometric contraction.

\section{Comparison of bilateral internal oblique muscle activity}

The TSP with HAB15 showed significantly greater bilateral IO muscle activity than TSP with $\operatorname{HAB} 0(p<0.05)$. In TSP with HAB30, bilateral IO muscle activity was significantly greater than TSP with HAB0 and TSP with HAB15 $(p<0.05)$. In OSP with HAB15, bilateral IO muscles activity was significantly greater than TSP with HAB0 and TSP with
HAB15 $(p<0.05)$. However, there was no significant difference compared with TSP with HAB30. In OSP with HAB30, TSP with HAB0, TSP with HAB15, OSP with HAB15 and OSP with HAB15 compared with TSP with HAB0, TSP with HAB15 and OSP with HAB15 $(p<0.05)$ (Figure 4, Table 2). 


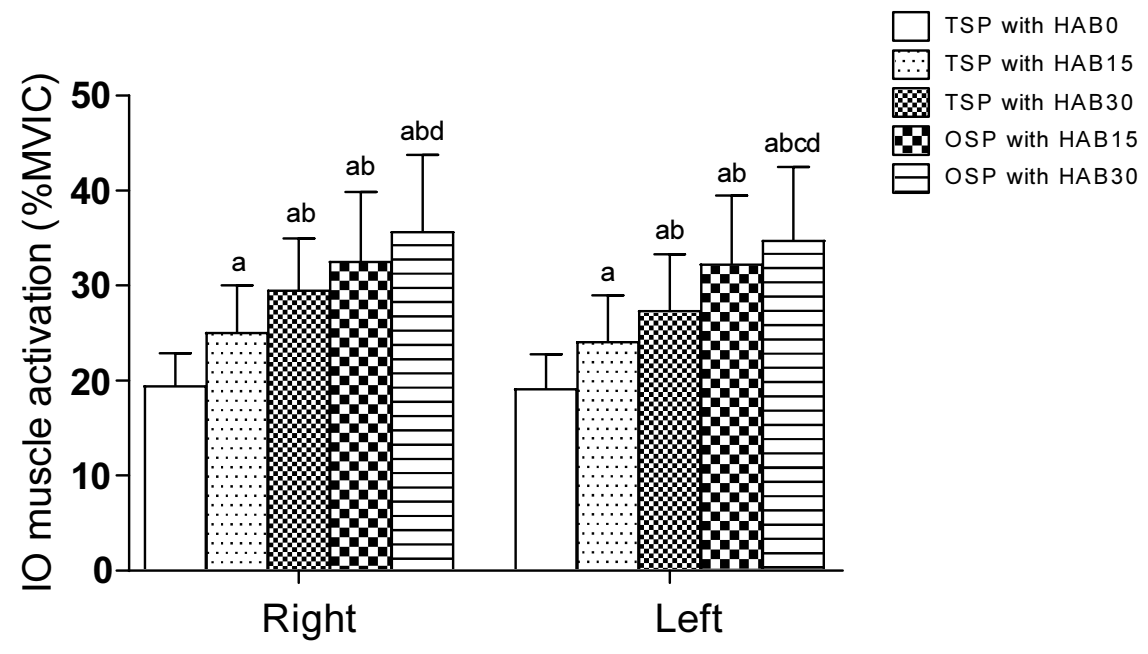

TSP=Two leg support plank; OSP=One leg support plank; $\mathrm{HAB}=$ =Hip abduction.

${ }^{\text {a }}$ Statistically significant difference with TSP with HABO $(p<0.05)$.

${ }^{\mathrm{b}}$ Statistically significant difference with TSP with HAB15 $(p<0.05)$.

'Statistically significant difference with TSP with HAB30 $(p<0.05)$.

${ }^{d}$ Statistically significant difference with OSP with HAB15 $(p<0.05)$.

Figure 4. Internal oblique (IO) muscle activities of 5 conditions. MVIC: maximum voluntary isometric contraction.

\section{Discussion}

The purpose of this study was to compare the muscle activity of the RA and the external and IO muscles using electromyography in order to investigate the effects of plank exercise on trunk muscle activity in healthy normal adults. The results of this study showed that the muscle activity during the OSP with hip abduction was greater than that during the normal plank motion.

Plank exercises are popular and beneficial in rehabilitation programs [17], and the abdominal muscles of the trunk are more active when compared to the trunk extensors [5]. The movement of the limbs, such as hip joint adduction and abduction, contributes to the transfer of force to the trunk muscles through the ASIS [7], and activation of the abdominal muscles with additional limb movements has been reported to occur with larger amplitudes when the predicted instability is applied to the trunk and when the activation patterns of the abdominal muscle is associated with the direction of limb movement [13,14,18].

In a study by Choi et al. [19], the activity of the internal and EO muscles were greater in the plank posture with hip joint abduction than in the basic plank exercise. Yoon et al. [20] reported that the muscle activities of bilateral RA and the IO were greater in the plank posture with hip abduction. Another study showed that plank exercise with hip isometric abduction contraction increased the activity of the internal and EO muscles more than the normal plank [21], and the EO muscle activity of the ipsilateral side was greater when the abduction of one hip occurred in the stance position [22].

The reason for the increase in the activity of the trunk muscle in the hip abduction appears to be related to the lever arm length of the muscle. The length of the lever arm optimizes muscle activation because hip abduction has made the lever arm larger, resulting in an increased need for mechanical use of the involved muscle [23].

Hip abduction also increases torque in the transverse plane that causes trunk and lumbar pelvic rotation and moves the center of mass of the body from the center to the outer side, and it can be stated that there is a compensatory increase in trunk muscle activity in order to maintain trunk balance [20].

Therefore, in this study, the plank motion with hip abduction exhibited greater increase in trunk muscle activity than the general plank motion. In addition, the results of the present study showed that the trunk muscle activity in the onelegged support plank was greater than the TSP with use of the same hip abduction angle.

To further increase the activation of trunk muscles, it is necessary to increase the level of instability [24-26]. It has been suggested that the use of dynamic cushioning on the toe or elbow with plank exercises to create instability contributes to a greater change in trunk muscle activation [9], there was greater RA and EO muscle activity in the plank with one 
leg suspended in a supporting device compared to the normal plank position [6], and greater anterior trunk muscle activity has been reported during plank exercises with the upper limbs placed on an unstable support surface compared to the normal plank [27]. This suggests that muscle activity was increased to compensate for instability caused by the reduction of the supporting surface with the floor [28].

During the plank exercise performed with the upper limb supported on the Swiss ball, the activity of the abdominal muscles was greater when one limb was lifted because the supporting surface of one limb was decreased and torque along the longitudinal axis of the body was generated in order to make compensations to maintain trunk balance [5].

In addition, the RA and the external and $\mathrm{IO}$ muscles showed greater activity when performing a one-legged bridge or a bridge on an unstable surface compared to the normal bridge because the trunk rotation strategy was selected in order to balance the trunk when the legs fell off the support surface [22]. There was greater RA and IO muscle activity during the OSP [20] since the lower fibers of the IO muscle is indirectly attached to the lumbar spine through the thoracic lumbar fascia, thereby increasing the stability of the lumbar spine and trunk with increased muscle activity [29,30], and since the RA muscle acts to stabilize and move the lumbar spine [30], and RA muscle activity was high in order to maintain the trunk in neutral position [20].

For the same reason in this study, the activity of the trunk muscles was greater in the OSP with reduced support surface than that of the TSP position.

There are some limitations to this study. First, due to a small sample size, it is difficult to generalize the findings to the normal, healthy population. In addition, since only three of the anterior trunk muscles were analyzed, other trunk muscles could not be confirmed, and long-term effect could not be seen using a cross-sectional study method. Also, the learning effect could not be avoided since the subjects were randomly assigned to five activities. Finally, it was not possible to quantify the extent to which the lumbar spine pelvis maintained its neutral position because the spinal pelvic alignment status of the subjects could not generalized. In future studies, it is necessary to conduct a training study to observe the long-term effects of exercise.

In conclusion, this study showed that there was a difference in trunk muscle activity according to various angles of hip abduction in healthy normal adults. Through this study, it is suggested that the plank exercises are effective for increasing trunk stability and can be customized according to the characteristics of the individual. In the following study, with consideration of the limitations and rather than a crosssectional research method, further studies on specific exercise methods that can be effectively applied to the subjects who require trunk stabilization exercises and aims to investigate the differences in muscle activity according to each individual's spinal pelvic alignment are needed.

\section{Conflict of Interest}

The authors declared no potential conflicts of interest with respect to the authorship and/or publication of this article.

\section{References}

1. Macedo LG, Saragiotto BT, Yamato TP, Costa LO, Menezes Costa LC, Ostelo RW, et al. Motor control exercise for acute non-specific low back pain. Cochrane Database Syst Rev 2016; 2:CD012085.

2. McGill SM, Cholewicki J. Biomechanical basis for stability: an explanation to enhance clinical utility. J Orthop Sports Phys Ther 2001;31:96-100.

3. Rabin A, Shashua A, Pizem K, Dickstein R, Dar G. A clinical prediction rule to identify patients with low back pain who are likely to experience short-term success following lumbar stabilization exercises: a randomized controlled validation study. J Orthop Sports Phys Ther 2014;44:6-B13.

4. Lehman GJ, Hoda W, Oliver S. Trunk muscle activity during bridging exercises on and off a Swiss ball. Chiropr Osteopat 2005; $13: 14$.

5. Escamilla RF, Lewis C, Bell D, Bramblet G, Daffron J, Lambert $\mathrm{S}$, et al. Core muscle activation during Swiss ball and traditional abdominal exercises. J Orthop Sports Phys Ther 2010;40:26576.

6. Kim SY, Kang MH, Kim ER, Jung IG, Seo EY, Oh JS. Comparison of EMG activity on abdominal muscles during plank exercise with unilateral and bilateral additional isometric hip adduction. J Electromyogr Kinesiol 2016;30:9-14.

7. Snarr RL, Esco MR. Electromyographical comparison of plank variations performed with and without instability devices. J Strength Cond Res 2014;28:3298-305.

8. Reed CA, Ford KR, Myer GD, Hewett TE. The effects of isolated and integrated 'core stability' training on athletic performance measures: a systematic review. Sports Med 2012;42:697-706.

9. Lee D, Lee Y, Cho HY, Lee KB, Hong S, Pyo S, et al. Investigation of trunk muscle activity for modified plank exercise: a preliminary study. Isokinet Exerc Sci 2017;25:209-13.

10. Peterson DD. Proposed performance standards for the plank for inclusion consideration into the navy's physical readiness test. Strength Cond J 2013;35:22-6.

11. Schoenfeld BJ, Contreras B, Tiryaki-Sonmez G, Willardson JM, Fontana F. An electromyographic comparison of a modified version of the plank with a long lever and posterior tilt versus the tra- 
ditional plank exercise. Sports Biomech 2014;13:296-306.

12. Mueller J, Hadzic M, Mugele H, Stoll J, Mueller S, Mayer F. Effect of high-intensity perturbations during core-specific sensorimotor exercises on trunk muscle activation. J Biomech 2018;70:212-8.

13. Lee DK, Kang MH, Kim JW, Kim YG, Park JH, Oh JS. Effects of non-paretic arm exercises using a tubing band on abdominal muscle activity in stroke patients. NeuroRehabilitation 2013;33: 605-10.

14. Hodges P, Cresswell A, Thorstensson A. Preparatory trunk motion accompanies rapid upper limb movement. Exp Brain Res 1999;124:69-79.

15. Criswell E. Cram's introduction to surface electromyography. Sudbury: Jones \& Bartlett Publishers; 2010.

16. Kendall FP, McCreary E, Provance P, Rodgers M, Romani W. Muscles: testing and function with posture and pain. Philadelphia: Lippincott Williams \& Wilkins; 2005.

17. D'Amico J, Betlach M, Senkarik R, Smith R, Voight M. Return to golf following left total hip arthroplasty in a golfer who is right handed. N Am J Sports Phys Ther 2007;2:251-61.

18. Aruin AS, Latash ML. Directional specificity of postural muscles in feed-forward postural reactions during fast voluntary arm movements. Exp Brain Res 1995;103:323-32.

19. Choi K, Bak J, Cho M, Chung Y. The effects of performing a one-legged bridge with hip abduction and use of a sling on trunk and lower extremity muscle activation in healthy adults. J Phys Ther Sci 2016;28:2625-8.

20. Yoon JO, Kang MH, Kim JS, Oh JS. Effect of modified bridge exercise on trunk muscle activity in healthy adults: a cross sectional study. Braz J Phys Ther 2018;22:161-7.

21. Kang MH, Kim SY, Kang MJ, Yoon SH, Oh JS. Effects of isometric hip movements on electromyographic activities of the trunk muscles during plank exercises. J Phys Ther Sci 2016;28: 2373-5.

22. Bhanot K, Kaur N, Brody LT, Bridges J, Berry DC, Ode JJ. Hip and trunk muscle activity during the star excursion balance test in healthy adults. J Sport Rehabil 2019;28:682-91.

23. Bolgla LA, Uhl TL. Electromyographic analysis of hip rehabilitation exercises in a group of healthy subjects. J Orthop Sports Phys Ther 2005;35:487-94.

24. Calatayud J, Borreani S, Martin J, Martin F, Flandez J, Colado JC. Core muscle activity in a series of balance exercises with different stability conditions. Gait Posture 2015;42:186-92.

25. Snarr RL, Hallmark AV, Nickerson BS, Esco MR. Electromyographical comparison of pike variations performed with and without instability devices. J Strength Cond Res 2016;30:343642.

26. Wolburg T, Rapp W, Rieger J, Horstmann T. Muscle activity of leg muscles during unipedal stance on therapy devices with different stability properties. Phys Ther Sport 2016;17:58-62.

27. Atkins SJ, Bentley I, Brooks D, Burrows MP, Hurst HT, Sinclair JK. Electromyographic response of global abdominal stabilizers in response to stable- and unstable-base isometric exercise. J Strength Cond Res 2015;29:1609-15.

28. Shumway-Cook A, Woollacott MH. Motor control: translating research into clinical practice. Philadelphia: Lippincott Williams \& Wilkins; 2007.

29. Kohler JM, Flanagan SP, Whiting WC. Muscle activation patterns while lifting stable and unstable loads on stable and unstable surfaces. J Strength Cond Res 2010;24:313-21.

30. Richardson C, Hodges PW, Hides J. Therapeutic exercise for lumbopelvic stabilization: a motor control approach for the treatment and prevention of low back pain. 2nd ed. Edinburgh U.K.: Churchill Livingstone; 2004. 